\title{
THE PSEUDO-HOPF BIFURCATION FOR PLANAR DISCONTINUOUS PIECEWISE LINEAR DIFFERENTIAL SYSTEMS
}

\author{
JUAN CASTILLO ${ }^{1}$, JAUME LLIBRE ${ }^{2}$ AND FERNANDO VERDUZCO ${ }^{1}$
}

\begin{abstract}
The creation or destruction of a crossing limit cycle when a sliding segment changes its stability, is known as pseudo-Hopf bifurcation. In this paper, under generic conditions, we find an unfolding for such bifurcation, and we prove the existence and uniqueness of a crossing limit cycle for this family.
\end{abstract}

\section{INTRODUCTION}

The study of limit cycles is one of the most important problem in the qualitative theory of ordinary differential equations, however, the proof of their existence are generally very complicated. A large list of papers about the arising of limit cycles in piecewise smooth systems in the plane can be found in the literature of recent years, and in these some techniques has been developed to find them. In smooth systems there is a well known mechanism to search for the occurrence of limit cycles, the Hopf bifurcation theorem, see $[13,19]$. There are analogous results for piecewise smooth systems, for the case of continuous systems see for example [6, 7, 26, 27], and for the case of discontinuous systems see $[1,8,11,12,14,18]$. In the discontinuous ones we can have more than one limit cycle, either all crossing cycles or including one sliding cycle, and in fact, the determination of the number of limit cycle has been the subject of several recent papers, see $[2,3,4,10,15,16,17,20,22,23,24]$.

When the appearance of more than one limit cycle is considered, often the mechanism to obtain one of them is by the collision of two invisible tangencies. This is, the creation or destruction of one crossing limit cycle occurs when a sliding segment changes its stability, this phenomenon is presented without demonstration in [18] and called pseudo-Hopf bifurcation. The appearance of a crossing limit cycle may occur in cases where there is not sliding segment, see $[9,21,25]$.

In this paper we find an unfolding for the pseudo-Hopf bifurcation for planar discontinuous piecewise linear (DPWL) systems with two zones separated by a straight line. We prove the existence and uniqueness of a crossing limit cycle for all possible dynamic scenarios. It is important to mention that the unfolding found has seven parameters, but at moment that the dynamics on each zone be established, it reduces to five. However, in our result it will not be necessary to establish a priori the dynamics in each zone.

2010 Mathematics Subject Classification. Primary 34A30, 34C05, 34C25, 34C07, 37G15.

Key words and phrases. Pseudo-Hopf bifurcation, discontinuous piecewise linear differential systems, sliding segment, unfolding. 
The rest of the paper is organized as follows. In section 2 we define the mathematical concepts used. In section 3 we state the main results. In section 4 we find the unfolding. The existence, uniqueness and stability of the crossing limit cycle is given in section 5. Finally, in section 6 we give the conclusions of this work.

\section{Preliminaries}

Consider the planar DPWL system with two zones separated by the straight line $\Sigma=\left\{x \in \mathbb{R}^{2}: \sigma(x)=c^{T} x-c_{0}=0\right\}$,

$$
\dot{x}=f(x)= \begin{cases}f^{-}(x)=A_{1} x+b_{1} & \text { if } \sigma(x)<0, \\ f^{+}(x)=A_{2} x+b_{2} & \text { if } \sigma(x)>0,\end{cases}
$$

where $A_{i}$ are $2 \times 2$ matrices and $b_{i} \in \mathbb{R}^{2}$ for $i=1,2$.

We distinguish three open regions in the straight line $\Sigma$ :

The sliding region: $\Sigma_{s}=\left\{x \in \Sigma: c^{T} f^{-}(x)>0\right.$ and $\left.c^{T} f^{+}(x)<0\right\}$, the escaping region: $\Sigma_{e}=\left\{x \in \Sigma: c^{T} f^{-}(x)<0\right.$ and $\left.c^{T} f^{+}(x)>0\right\}$, and the crossing region: $\Sigma_{c}=\left\{x \in \Sigma:\left(c^{T} f^{-}(x)\right)\left(c^{T} f^{+}(x)\right)>0\right\}$.

Any segment contained in $\Sigma_{s} \cup \Sigma_{e}$ is called a sliding segment. The solutions on $\Sigma_{s} \cup \Sigma_{e}$ can be constructed by the Filippov's convex method, see [5]. Filippov's method takes a simple convex combination $f_{s}(x)$ of the two vector fields $f^{\mp}(x)$ to each sliding point $x \in \Sigma_{s} \cup \Sigma_{e}$, i.e.

$$
f_{s}(x)=\frac{\tilde{f}_{s}(x)}{\Delta(x)},
$$

where $\tilde{f}_{s}(x)=\left(c^{T} f^{-}(x)\right) f^{+}(x)-\left(c^{T} f^{+}(x)\right) f^{-}(x)$ and $\Delta(x)=c^{T}\left(f^{-}(x)-f^{+}(x)\right) \neq$ 0 . $f_{s}$ is called the sliding vector field, while $\tilde{f}_{s}$ is called the regularized sliding vector field.

A point $x \in \mathbb{R}^{2}$ is an equilibrium point of $f^{-}$if $f^{-}(x)=0$ and $\sigma(x)<0$. The equilibrium point is virtual for $f^{-}$if $f^{-}(x)=0$ and $\sigma(x)>0$.

A point $\tilde{x} \in \Sigma$ is a pseudo-equilibrium of (1) if $f_{s}(\tilde{x})=0$. The pseudo-equilibrium is admissible if $\tilde{x} \in \Sigma_{s} \cup \Sigma_{e}$, or virtual if $\tilde{x} \in \Sigma_{c}$.

A point $\hat{x}$ is a boundary equilibrium of (1) if

$$
f^{-}(\hat{x}) f^{+}(\hat{x})=0 \text {, and } f_{s}(\hat{x})=0 .
$$

Since the three kinds of regions in $\Sigma$ are relatively open, their boundaries are the called tangency points: $q \in \Sigma$ such that $c^{T} f^{-}(q)=0$ or $c^{T} f^{+}(q)=0$ (see $\left.[12,18]\right)$. That is, points where one of the two vector fields is tangent to $\Sigma$. In particular, the boundary equilibria are tangency points, since they are located on the boundary of the sliding region where one of the vector fields vanishes. The simplest tangency is the fold singularity, which is defined as follows.

A point $q \in \Sigma$ is a fold singularity of (1) if either

(i) $c^{T} f^{-}(q)=0$ and $c^{T} A_{1} f^{-}(q) \neq 0$, or

(ii) $c^{T} f^{+}(q)=0$ and $c^{T} A_{2} f^{+}(q) \neq 0$, or

(iii) $q$ is a hyperbolic focus of $f^{-}$or $f^{+}$. 
A fold singularity is a point with quadratic tangency with $\Sigma$, or is a boundary focus. A quadratic tangency point can be classified in visible or invisible as follows:

(i) $q \in \Sigma$ is an invisible (visible) quadratic tangency point for $f^{-}$if

$$
c^{T} f^{-}(q)=0 \text { and } r_{1}=c^{T} A_{1} f^{-}(q)>0(<0) .
$$

(ii) $q \in \Sigma$ is an invisible (visible) quadratic tangency point for $f^{+}$if

$$
c^{T} f^{+}(q)=0 \text { and } r_{2}=c^{T} A_{2} f^{+}(q)<0(>0) .
$$

The case where system (1) has a quadratic tangency point for one vector field, and a boundary focus for the other one, at the same point on the switching line is called fold-focus singularity. When system (1) has a double boundary focus at the same point on the switching line, that is, when there is a boundary focus for both sides, this singularity is called the focus-focus singularity. Finally, a fold-fold singularity is when the DPWL system (1) has a double quadratic tangecy at the same point on $\Sigma$.

For the case of the invisible fold-fold singularity, when the vectors $f^{-}\left(q_{0}\right)$ and $f^{+}\left(q_{0}\right)$ are antiparallel with $q_{0} \in \partial \Sigma_{c}$, the singularity is called fused-focus in [18]. We are going to call two-fold singularity to the fold-fold, fold-focus or focus-focus singularities.

\section{Statements of the main Results}

The idea is to unfold the two-fold singularity $q_{0}$ in such way that two fold points, $q_{1}$ and $q_{2}$, from $f^{-}$and $f^{+}$, respectively, delimit a sliding segment, and when they change their relative position on $\Sigma$, after collapse in $q_{0}$, the sliding segment change its stability. As will be proved in this article, for some configurations of the fold points, this change of stability in the sliding segment is accompanied by the birth or destruction of a crossing limit cycle. See Figure 1. With this idea, we assume that $f(x)$ satisfy the following generic hypothesis:

$\left(H_{0}\right)$ The pairs of vectors $\left\{c, A_{1}^{T} c\right\}$ and $\left\{c, A_{2}^{T} c\right\}$ are linearly independent.
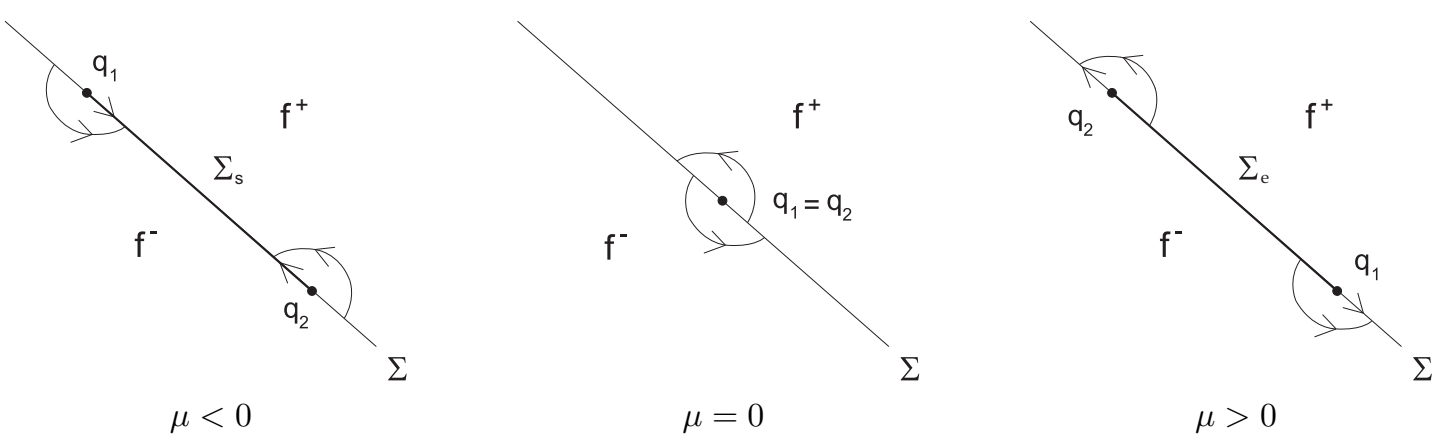

Figure 1. Change of stability of the sliding segment for the case of two invisible fold points. 
Under Hypothesis $\left(H_{0}\right)$, the DPWL system (1) has two fold points $q_{1}$ and $q_{2}$. This is clear because of, if we define the straight lines

$$
L_{i}: c^{T}\left(A_{i} x+b_{i}\right)=0, \text { then } L_{i} \cap \Sigma=\left\{q_{i}\right\},
$$

for $i=1,2$. Besides there exist $\gamma_{1}$ and $\gamma_{2}$ with $\gamma_{2} \neq 0$ such that

$$
A_{2}^{T} c=\gamma_{1} c+\gamma_{2} A_{1}^{T} c .
$$

The first theorem in this section give us an unfolding for piecewise linear systems that satisfy the generic hypothesis $\left(H_{0}\right)$.

Theorem 1. Under hypothesis $\left(H_{0}\right)$ the change of coordinates

$$
y=h(x)=\left\{\begin{array}{cc}
\gamma_{2} Q_{1}\left(x-q_{1}\right) & \text { if } \quad \sigma(x) \leq 0, \\
Q_{2}\left(x-q_{1}\right) & \text { if } \quad \sigma(x) \geq 0,
\end{array}\right.
$$

where $Q_{1}=\left(\begin{array}{c}c^{T} \\ c^{T} A_{1}\end{array}\right)$ and $Q_{2}=\left(\begin{array}{c}c^{T} \\ c^{T} A_{2}\end{array}\right)$, transforms the differential system (1) into the differential system

(3) $\dot{y}=F(y)= \begin{cases}F^{-}(y)=\left(\begin{array}{cc}0 & 1 \\ c_{1} & c_{2}\end{array}\right) y+\left(\begin{array}{c}0 \\ \gamma_{2} r_{1}\end{array}\right) & \text { if } y_{1}<0, \\ F^{+}(y)=\left(\begin{array}{cc}0 & 1 \\ d_{1} & d_{2}\end{array}\right) y+\left(\begin{array}{c}b \\ r_{2}+d_{2} b\end{array}\right) & \text { if } \quad y_{1}>0,\end{cases}$

where

$\begin{array}{lll}c_{1}=-\operatorname{det}\left(A_{1}\right), & c_{2}=\operatorname{trace}\left(A_{1}\right), & d_{1}=-\operatorname{det}\left(A_{2}\right), \\ d_{2}=\operatorname{trace}\left(A_{2}\right), & r_{1}=c^{T} A_{1}\left(A_{1} q_{1}+b_{1}\right), & r_{2}=c^{T} A_{2}\left(A_{2} q_{2}+b_{2}\right), \\ b=c^{T}\left(A_{2} q_{1}+b_{2}\right) & & \end{array}$

Theorem 1 is proved in section 4 .

Remark 1. (a) If $q_{2} \rightarrow q_{1}$ then $b \rightarrow 0$, i.e. at $b=0$, the fold points collapse at $q_{0}$. (b) If $r_{j}=0$ then the fold point $q_{j}$ is a boundary equilibrium point, which must be a boundary focus, with eigenvalues $\alpha_{j} \pm i \beta_{j}$ for $j=1,2$.

(c) If $r_{1}>0$ then $q_{1}$ is an invisible fold point.

(d) If $r_{2}<0$ then $q_{2}$ is an invisible fold point.

The following corollary establishes the $\Sigma$-equivalence of the change of coordinates (2), see [12].

Corollary 1. If $\gamma_{2}>0$ then $h\left(\Sigma_{a}\right)=\bar{\Sigma}_{a}$ for $a \in\{s, e, c\}$.

Proof. For $x \in \Sigma$ we have $h(x)=\left(\begin{array}{c}0 \\ \gamma_{2} c^{T} A_{1}\left(x-q_{1}\right)\end{array}\right)=\left(\begin{array}{c}0 \\ c^{T} A_{2}\left(x-q_{1}\right)\end{array}\right)$. Then

$$
\begin{aligned}
e_{1}^{T} F^{-}(h(x)) & =(1,0)\left(\begin{array}{c}
\gamma_{2} c^{T} A_{1}\left(x-q_{1}\right) \\
(\cdot)
\end{array}\right) \\
& =\gamma_{2} c^{T} A_{1}\left(x-q_{1}\right)+\gamma_{2} c^{T} b_{1}-\gamma_{2} c^{T} b_{1} \\
& =\gamma_{2} c^{T} f^{-}(x),
\end{aligned}
$$


and

$$
\begin{aligned}
e_{1}^{T} F^{+}(h(x)) & =(1,0)\left(\begin{array}{c}
c^{T} A_{2}\left(x-q_{1}\right)+b \\
(\cdot)
\end{array}\right) \\
& =c^{T} A_{2}\left(x-q_{1}\right)+c^{T}\left(A_{2} q_{1}+b_{2}\right) \\
& =c^{T} f^{+}(x) .
\end{aligned}
$$

Remark 2. The change of coordinates (2) classifies all the DPWL systems (1) that satisfy $\left(H_{0}\right)$ into two classes: those systems that have a sliding segment $\left(\gamma_{2}>0\right)$ and those that have a crossing segment $\left(\gamma_{2}<0\right)$.

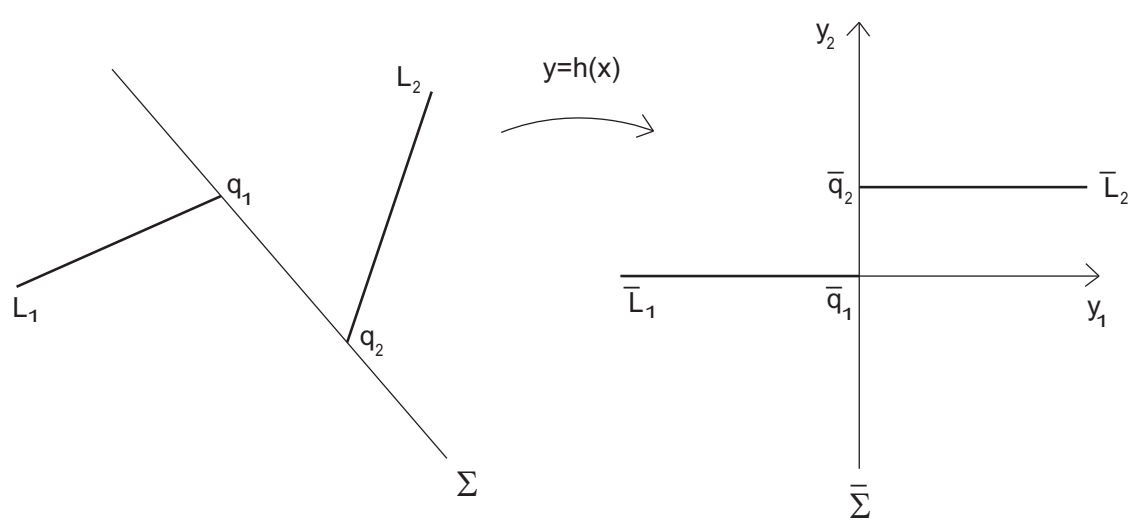

Figure 2. Change of coordinates (2).

From now on we will assume that $\gamma_{2}>0$. Figure 2 shows the effect of the orthogonalization of the change of coordinates (2). From the unfolding (3), for $b=0$, we find nine different scenarios in which the two-fold singularity can be unfolded in a such way that is it possible to observe a change of stability in a sliding segment. See Figure 3.

The main theorem of the paper establishes that the unfolding (3) undergoes the pseudo-Hopf bifurcation only at four cases $\left(r_{1} \geq 0\right.$ and $\left.r_{2} \leq 0\right)$.

Theorem 2 (Pseudo-Hopf bifurcation theorem). Suppose that the DPWL system (1) satisfy $\left(H_{0}\right)$ with $\gamma_{2}>0$. If $r_{1} \geq 0$ and $r_{2} \leq 0$, then for each $b$ sufficiently small with $b \Lambda_{0}<0$, system (1) has a unique crossing limit cycle. If $\Lambda_{0}<0$ the limit cycle is stable, while if $\Lambda_{0}>0$ it is unstable, where

$$
\Lambda_{0}=\left\{\begin{array}{ccc}
\frac{c_{2}}{\gamma_{2} r_{1}}-\frac{d_{2}}{r_{2}} & \text { if } & r_{1}>0, r_{2}<0 \text { (fused-focus), } \\
\alpha_{2} & \text { if } & r_{1}>0, r_{2}=0 \text { (fold-focus), } \\
\alpha_{1} & \text { if } & r_{1}=0, r_{2}<0 \text { (fold-focus), } \\
\frac{\alpha_{1}}{\beta_{1}}+\frac{\alpha_{2}}{\beta_{2}} & \text { if } & r_{1}=0, r_{2}=0 \text { (focus-focus). }
\end{array}\right.
$$




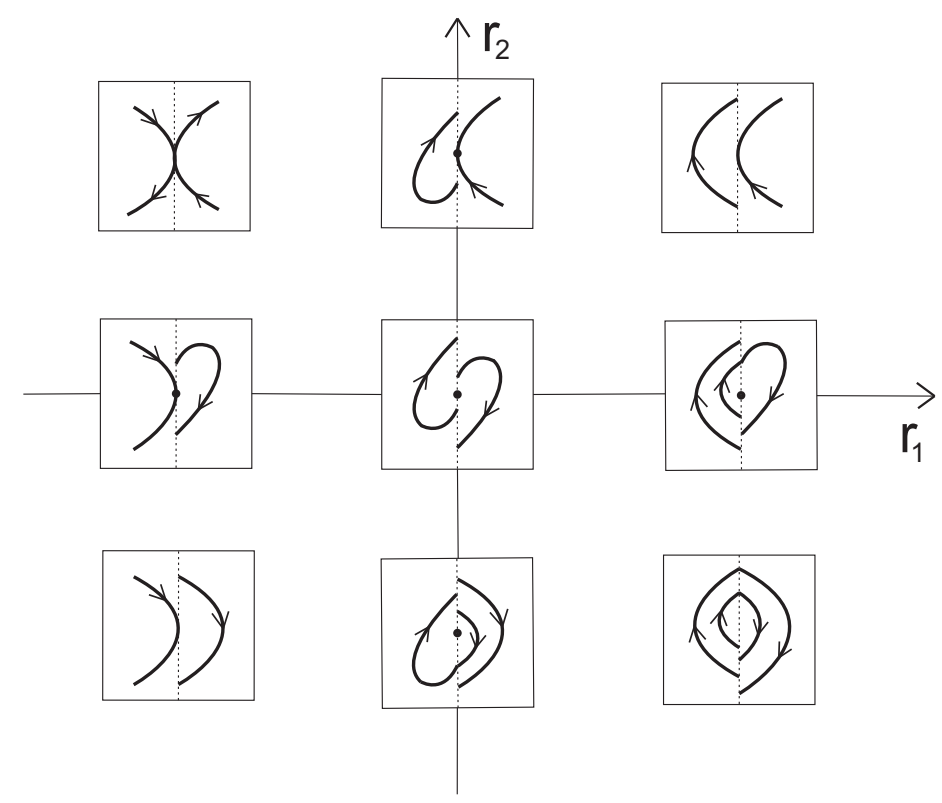

Figure 3 . The two-fold singularity for the unfolding (3).

Theorem 2 is proved in section 5 .

Remark 3. It is not necessary to calculate the change of coordinates (2), nor the unfolding (3) to use Theorem 2, it is enough to calculate the expressions given in (4) from the original DPWL system (1).

\section{Proof of Theorem 1}

For $x \in \Sigma$ we have

$$
h(x)=\left\{\begin{array}{c}
\gamma_{2} Q_{1}\left(x-q_{1}\right)=\left(\begin{array}{c}
\gamma_{2} c^{T}\left(x-q_{1}\right) \\
\gamma_{2} c^{T} A_{1}\left(x-q_{1}\right)
\end{array}\right)=\left(\begin{array}{c}
0 \\
c^{T} A_{2}\left(x-q_{1}\right)
\end{array}\right) \quad \text { if } \quad \sigma(x) \leq 0, \\
Q_{2}\left(x-q_{1}\right)=\left(\begin{array}{c}
c^{T}\left(x-q_{1}\right) \\
c^{T} A_{2}\left(x-q_{1}\right)
\end{array}\right)=\left(\begin{array}{c}
0 \\
c^{T} A_{2}\left(x-q_{1}\right)
\end{array}\right) \quad \text { if } \quad \sigma(x) \geq 0 .
\end{array}\right.
$$

That is, $h$ sends $\Sigma$ on $y_{1}=0$. For $\sigma(x) \leq 0$ we have $\dot{y}=F^{-}(y)=\bar{A}_{1} y+\bar{b}_{1}$, where

$$
\bar{A}_{1}=Q_{1} A_{1} Q_{1}^{-1}=\left(\begin{array}{c}
c^{T} A_{1} Q_{1}^{-1} \\
c^{T} A_{1}^{2} Q_{1}^{-1}
\end{array}\right)=\left(\begin{array}{cc}
0 & 1 \\
c_{1} & c_{2}
\end{array}\right),
$$

because of

$$
Q_{i} Q_{i}^{-1}=\left(\begin{array}{c}
c^{T} Q_{i}^{-1} \\
c^{T} A_{i} Q_{i}^{-1}
\end{array}\right)=I, \text { for } i=1,2
$$

and

$$
\bar{b}_{1}=\gamma_{2} Q_{1}\left(A_{1} q_{1}+b_{1}\right)=\left(\begin{array}{c}
0 \\
\gamma_{2} r_{1}
\end{array}\right) \text {. }
$$


For $\sigma(x) \geq 0$ we have $\dot{y}=F^{+}(y)=\bar{A}_{2} y+\bar{b}_{2}$, where

$$
\bar{A}_{2}=Q_{2} A_{2} Q_{2}^{-1}=\left(\begin{array}{c}
c^{T} A_{2} Q_{2}^{-1} \\
c^{T} A_{2}^{2} Q_{2}^{-1}
\end{array}\right)=\left(\begin{array}{cc}
0 & 1 \\
d_{1} & d_{2}
\end{array}\right)
$$

and

$$
\bar{b}_{2}=Q_{2}\left(A_{2} q_{1}+b_{2}\right)=\left(\begin{array}{c}
c^{T}\left(A_{2} q_{1}+b_{2}\right) \\
\left.c^{T} A_{2}\left(A_{2} q_{1}+b_{2}\right)\right)
\end{array}\right)=\left(\begin{array}{c}
b \\
\tilde{b}
\end{array}\right) .
$$

If $Q_{2}^{-1}=\left(\begin{array}{ll}v_{1} & v_{2}\end{array}\right)$ then $Q_{2} Q_{2}^{-1}=\left(\begin{array}{c}c^{T} \\ c^{T} A_{2}\end{array}\right)\left(v_{1} v_{2}\right)=I$. Observe that it is possible to normalize $c$ such that $\left\|v_{2}\right\|=1$. We define $v=q_{2}-q_{1}$, then

$\tilde{b}=c^{T} A_{2}\left(A_{2} q_{1}+b_{2}\right)=c^{T} A_{2}\left(A_{2}\left(q_{2}-v\right)+b_{2}\right)=c^{T} A_{2}\left(A_{2} q_{2}+b_{2}\right)-c^{T} A_{2}^{2} v=r_{2}-c^{T} A_{2}^{2} v$.

Besides there exist $s_{1}, s_{2} \in \mathbb{R}$ such that $v=s_{1} v_{1}+s_{2} v_{2}$, but $0=c^{T} v=s_{1} c^{T} v_{1}+$ $s_{2} c^{T} v_{2}=s_{1}$, then

$$
v=s_{2} v_{2} .
$$

Therefore $c^{T} A_{2}^{2} v=s_{2} c^{T} A_{2}^{2} v_{2}=s_{2} d_{2}$, and $c^{T} A_{2} v=s_{2} c^{T} A_{2} v_{2}=s_{2}$, that is

(6) $s_{2}=c^{T} A_{2} v=c^{T} A_{2}\left(q_{2}-q_{1}\right)+c^{T} b_{2}-c^{T} b_{2}=-c^{T}\left(A_{2} q_{1}+b_{2}\right)=-b$.

This completes the proof of theorem 1 .

Remark 4. From (5) and (6) it follows that $\left\|q_{2}-q_{1}\right\|=|b|$.

\section{Proof of Theorem 2}

Consider the unfolding (3), i.e.

$$
\dot{y}= \begin{cases}\left(\begin{array}{cc}
0 & 1 \\
c_{1} & c_{2}
\end{array}\right) y+\left(\begin{array}{c}
0 \\
\gamma_{2} r_{1}
\end{array}\right) & \text { if } \quad y_{1}<0, \\
\left(\begin{array}{cc}
0 & 1 \\
d_{1} & d_{2}
\end{array}\right) y+\left(\begin{array}{c}
b \\
r_{2}+d_{2} b
\end{array}\right) \text { if } \quad y_{1}>0 . & \end{cases}
$$

We call $\phi_{t}$ and $\psi_{t}$ the flow for $y_{1}<0$ and $y_{1}>0$, respectively. To prove the existence of a crossing limit cycle we are going to find $\hat{q}_{1}=\left(\begin{array}{l}0 \\ u\end{array}\right)$ and $\hat{q}_{2}=\left(\begin{array}{l}0 \\ v\end{array}\right)$, with $u>0$ and $v<0$, and times $t_{1}, t_{2}$, such that the system

$$
\begin{aligned}
& S_{1}=\phi_{t_{1}}\left(\hat{q}_{2}\right)-\hat{q}_{1}=0, \\
& S_{2}=\psi_{t_{2}}\left(\hat{q}_{1}\right)-\hat{q}_{2}=0,
\end{aligned}
$$

has a unique solution. See Figure 4.

We rename $A_{1}=\left(\begin{array}{cc}0 & 1 \\ c_{1} & c_{2}\end{array}\right)$ and $A_{2}=\left(\begin{array}{cc}0 & 1 \\ d_{1} & d_{2}\end{array}\right)$, and consider $\sigma\left(A_{1}\right)=$ $\left\{\lambda_{1}, \lambda_{2}\right\}$ and $\sigma\left(A_{2}\right)=\left\{\delta_{1}, \delta_{2}\right\}$.

5.1. Fused-focus singularity: $r_{1}>0$ and $r_{2}<0$. 


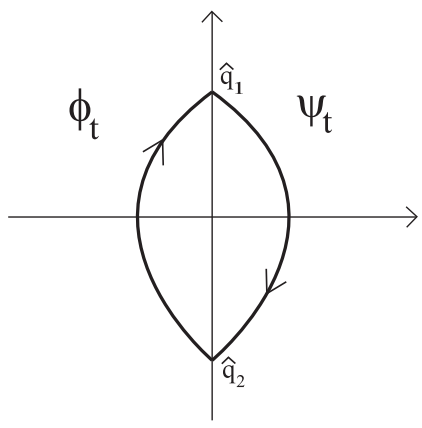

Figure 4. A crossing limit cycle.

5.1.1. Case $\lambda_{1} \lambda_{2} \neq 0, \lambda_{1} \neq \lambda_{2}, \delta_{1} \delta_{2} \neq 0, \delta_{1} \neq \delta_{2}$. In this case

$$
S_{1}=0 \Leftrightarrow\left\{\begin{array}{l}
e^{\lambda_{1} t_{1}}=\frac{\gamma_{2} r_{1}+\lambda_{1} u}{\gamma_{2} r_{1}+\lambda_{1} v}=\frac{1+\tilde{\lambda}_{1} u}{1+\tilde{\lambda}_{1} v}, \\
e^{\lambda_{2} t_{1}}=\frac{\gamma_{2} r_{1}+\lambda_{2} u}{\gamma_{2} r_{1}+\lambda_{2} v}=\frac{1+\tilde{\lambda}_{2} u}{1+\tilde{\lambda}_{2} v}
\end{array}\right.
$$

where $\tilde{\lambda}_{i}=\frac{\lambda_{i}}{\gamma_{2} r_{1}}$. Observe that for $u>0, v<0$, and $\tilde{\lambda}_{i} \in \mathbb{R}$ we have

$$
\frac{1+\tilde{\lambda}_{i} u}{1+\tilde{\lambda}_{i} v}>0 \Leftrightarrow 1+\tilde{\lambda}_{i} u>0 \text { and } 1+\tilde{\lambda}_{i} v, \text { for } i=1,2
$$

Therefore $e^{\lambda_{i} t_{1}}=\frac{1+\tilde{\lambda}_{i} u}{1+\tilde{\lambda}_{i} v} \Leftrightarrow e^{t_{1}}=\left(\frac{1+\tilde{\lambda}_{i} u}{1+\tilde{\lambda}_{i} v}\right)^{\frac{1}{\lambda_{i}}}=\frac{\left(1+\tilde{\lambda}_{i} u\right)^{\frac{1}{\lambda_{i}}}}{\left(1+\tilde{\lambda}_{i} v\right)^{\frac{1}{\lambda_{i}}}}$, then

$$
S_{1}=0 \Leftrightarrow G_{1}(u, v)=\frac{\left(1+\tilde{\lambda}_{1} u\right)^{\frac{1}{\lambda_{1}}}}{\left(1+\tilde{\lambda}_{2} u\right)^{\frac{1}{\lambda_{2}}}}-\frac{\left(1+\tilde{\lambda}_{1} v\right)^{\frac{1}{\lambda_{1}}}}{\left(1+\tilde{\lambda}_{2} v\right)^{\frac{1}{\lambda_{2}}}}=0 .
$$

Similarly

$$
\begin{array}{r}
S_{2}=0 \Leftrightarrow\left\{\begin{array}{l}
e^{\delta_{1} t_{2}}=\frac{r_{2}+\delta_{1}(v+b)}{r_{2}+\delta_{1}(u+b)}=\frac{1+\tilde{\delta}_{1}(-v-b)}{1+\tilde{\delta}_{1}(-u-b)}, \\
e^{\delta_{2} t_{2}}=\frac{r_{2}+\delta_{2}(v+b)}{r_{2}+\delta_{2}(u+b)}=\frac{1+\tilde{\delta}_{2}(-v-b)}{1+\tilde{\delta}_{2}(-u-b)},
\end{array}\right. \\
\Leftrightarrow G_{2}(u, v)=\frac{\left(1+\tilde{\delta}_{1}(-v-b)\right) \frac{1}{\delta_{1}}}{\left(1+\tilde{\delta}_{2}(-v-b)\right)^{\frac{1}{\delta_{2}}}}-\frac{\left(1+\tilde{\delta}_{1}(-u-b)\right)}{\left(1+\tilde{\delta}_{2}(-u-b)\right) \frac{1}{\delta_{2}}}=0,
\end{array}
$$




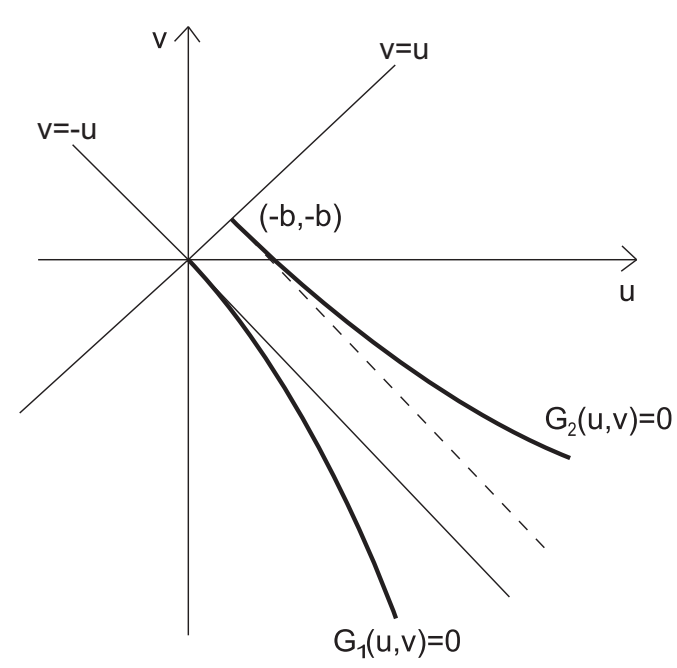

FiguRE 5. Curve solutions for system $S_{1}=S_{2}=0$.

where $\tilde{\delta}_{i}=-\frac{\delta_{i}}{r_{2}}$. Observe that $G_{1}(0,0)=0$ and, for $b=0, G_{2}(0,0)=0$. Moreover if $\lambda_{i}=\delta_{i}$ and $\frac{1}{\gamma_{2} r_{1}}=-\frac{1}{r_{2}}$, then $\tilde{\lambda}_{i}=\tilde{\delta}_{i}$ for $i=1,2$, and

$$
G_{2}(u, v)=G_{1}(-(v+b),-(u+b)) .
$$

That is, the curve $G_{2}(u, v)=0$ can be obtained by the reflection of the curve $G_{1}(u, v)=0$, with respect to the straight line $v=-u$ followed by the translation $(-b,-b)^{T}$. See Figure 5. Then it is sufficient to solve $G_{1}(u, v)=0$.

Lemma 1. There exists a smooth function $h:\left(-\varepsilon_{1}, 0\right] \rightarrow\left[0, \varepsilon_{2}\right)$ such that $G_{1}(h(v), v)=$ 0 for each $v \in\left(-\varepsilon_{1}, 0\right]$. Furthermoe $h(0)=0, h^{\prime}(0)=-1$ and $h^{\prime \prime}(0)=\frac{4 c_{2}}{3 \gamma_{2} r_{1}}$.

Proof. First we observe that

$$
G_{1}(u, v)=0 \Leftrightarrow H(u)=H(v),
$$

where $H(z)=\frac{\left(1+\tilde{\lambda}_{1} z\right)^{\frac{1}{\lambda_{1}}}}{1}$. We are going to distinguish two cases.

$$
\left(1+\tilde{\lambda}_{2} z\right)^{\frac{1}{\lambda_{2}}}
$$

Real case: $\lambda_{1}, \lambda_{2} \in \mathbb{R}$. In this case $H: \mathbb{R} \rightarrow \mathbb{R}$, and after some calculations $H(0)=1, H^{\prime}(0)=0$, and $H^{\prime \prime}(0)=\frac{\lambda_{2}-\lambda_{1}}{\left(\gamma_{2} r_{1}\right)^{2}}>0$, then $H$ has a local minimum at $z=0$. Then there exist $\varepsilon_{1}, \varepsilon_{2}>0$ such that for each $v \in\left(-\varepsilon_{1}, 0\right)$ there exists a unique $u \in\left(0, \varepsilon_{2}\right)$ with $H(u)=H(v)$. That is, there exists a function $h:\left(-\varepsilon_{1}, 0\right] \rightarrow\left[0, \varepsilon_{2}\right)$ such that $h(0)=0$ and

$$
H(v)=H(h(v)) \Leftrightarrow G_{1}(h(v), u)=0,
$$


for each $v \in\left(-\varepsilon_{1}, 0\right]$. See Figure 6 .

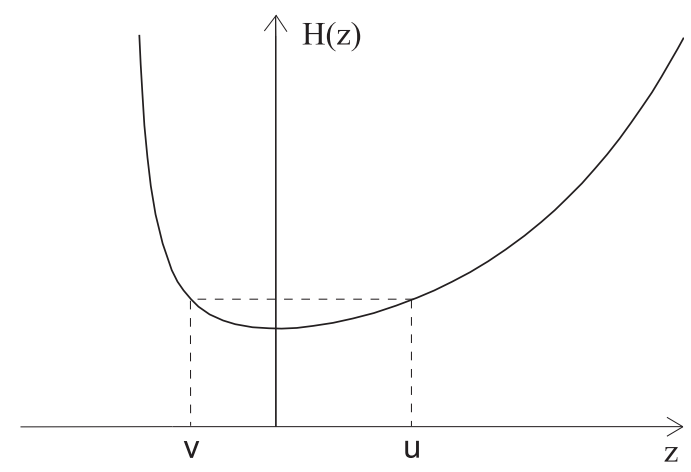

FigURE 6. $u=h(v)$

From $H^{\prime}(v)=H^{\prime}(h(v)) h^{\prime}(v)$ we get

$$
h^{\prime}(v)=\frac{H^{\prime}(v)}{H^{\prime}(h(v))}<0,
$$

for each $v \in\left(-\varepsilon_{1}, 0\right]$, because of $H^{\prime}(v)<0$ and $H^{\prime}(h(v))>0$. If we assume that $h^{\prime}(0)$ exists, then

$h^{\prime}(0)=\lim _{v \rightarrow 0^{-}} h^{\prime}(v)=\lim _{v \rightarrow 0^{-}} \frac{H^{\prime}(v)}{H^{\prime}(h(v))}=\lim _{v \rightarrow 0^{-}} \frac{H^{\prime \prime}(v)}{H^{\prime \prime}(h(v)) h^{\prime}(v)}=\frac{1}{h^{\prime}(0)} \Leftrightarrow h^{\prime}(0)=-1$.

Therefore from $H^{\prime \prime}(v)=H^{\prime \prime}(h(v))\left(h^{\prime}(v)\right)^{2}+H^{\prime}(h(v)) h^{\prime \prime}(v)$ we obtain

$$
h^{\prime \prime}(v)=\frac{H^{\prime \prime}(v)-H^{\prime \prime}(h(v))\left(h^{\prime}(v)\right)^{2}}{H^{\prime}(h(v))},
$$

and again, if we assume that $h^{\prime \prime}(0)$ exists, then

$$
\begin{aligned}
& h^{\prime \prime}(0)=\lim _{v \rightarrow 0^{-}} h^{\prime \prime}(v)=\lim _{v \rightarrow 0^{-}} \frac{H^{\prime \prime}(v)-H^{\prime \prime}(h(v))\left(h^{\prime}(v)\right)^{2}}{H^{\prime}(h(v))} \\
& =\lim _{v \rightarrow 0^{-}} \frac{H^{\prime \prime \prime}(v)-H^{\prime \prime \prime}(h(v))\left(h^{\prime}(v)\right)^{3}-2 H^{\prime \prime}(h(v)) h^{\prime}(v) h^{\prime \prime}(v)}{H^{\prime \prime}(h(v)) h^{\prime}(v)} \\
& =-\frac{2 H^{\prime \prime \prime}(0)}{H^{\prime \prime}(0)}-2 h^{\prime \prime}(0),
\end{aligned}
$$

Complex case: $\lambda_{1}=\lambda=\alpha+\beta i \in \mathbb{C}$ with $\beta>0$. In this case $H: \mathbb{R} \rightarrow \mathbb{C}$. It is known that for $z_{1}, z_{2} \in \mathbb{C}$ we have $\overline{z_{1}^{z_{2}}}=\bar{z}_{1}^{\bar{z}_{2}}$. Therefore if $w(z)=(1+\tilde{\lambda} z)^{\frac{1}{\lambda}}=$ $r(z) e^{i \theta(z)}$, then

$$
H(z)=\frac{w(z)}{\bar{w}(z)}=e^{2 i \theta(z)} .
$$


That is

$$
G_{1}(u, v)=0 \Leftrightarrow H(u)=H(v) \Leftrightarrow \theta(u)=\theta(v) .
$$

We are going to find the real function $\theta(z)$. If $\tilde{\lambda}=\frac{1}{\gamma_{2} r_{1}}(\lambda)=\tilde{\alpha}+\tilde{\beta} i$, then $1+\tilde{\lambda} z=(1+\tilde{\alpha} z)+i(\tilde{\beta} z)=r_{0}(z) e^{i \theta_{0}(z)}$. Therefore

$$
\begin{aligned}
(1+\tilde{\lambda} z)^{\frac{1}{\lambda}}=r(z) e^{i \theta(z)} & \Leftrightarrow \operatorname{Ln}\left(r_{0} e^{i \theta_{0}}\right)=\lambda \operatorname{Ln}\left(r e^{i \theta}\right) \\
& \Leftrightarrow\left(\alpha \operatorname{Ln}(r)-\operatorname{Ln}\left(r_{0}\right)-\beta \theta\right)+i\left(\alpha \theta+\beta \operatorname{Ln}(r)-\theta_{0}\right)=0 .
\end{aligned}
$$

Then

$$
\theta(z)=\frac{1}{|\lambda|^{2}}\left(\alpha \arctan \left(\frac{\tilde{\beta} z}{1+\tilde{\alpha} z}\right)-\frac{\beta}{2} \operatorname{Ln}\left((1+\tilde{\alpha} z)^{2}+(\tilde{\beta} z)^{2}\right)\right)
$$

After some calculations, we find that $\theta(0)=\theta^{\prime}(0)=0$, and $\theta^{\prime \prime}(0)=-\frac{\beta}{\gamma_{2} r_{1}}<0$, then $\theta$ has a local maximum at $z=0$. Following the same arguments than in the real case we prove that there exists a real function $u=h(v)$ such that $G_{1}(h(v), v)=0$, for each $v \in\left(-\varepsilon_{1}, 0\right]$, where $h(0)=0, h^{\prime}(0)=-1$, and $h^{\prime \prime}(0)=\frac{4}{3 \gamma_{2} r_{1}}(\lambda+\bar{\lambda})$. This completes the proof of the lemma.

Existence and stability. From Lemma 1 and (9) it follows that the solutions of system $(7-8), h_{1}(v)$ and $h_{2}(u)$ are given by

$$
\begin{aligned}
& u=h_{1}(v)=-v+\lambda_{0} v^{2}-\lambda_{0}^{2} v^{3}+\cdots, \\
& v=h_{2}(u)=-2 b-u-\delta_{0}(b+u)^{2}-\delta_{0}^{2}(b+u)^{3}+\cdots,
\end{aligned}
$$

where $\lambda_{0}=\frac{2 c_{2}}{3 \gamma_{2} r_{1}}$ and $\delta_{0}=-\frac{2 d_{2}}{3 r_{2}}$. For $\varepsilon>0$ we define the Poincaré map $P$ : $(-\varepsilon, 0) \rightarrow(-\varepsilon, 0)$ given by

$$
\begin{aligned}
P(v, b) & =h_{2}\left(h_{1}(v)\right)=v-\left(\lambda_{0}+\delta_{0}\right) v^{2}+\mathcal{O}\left(|v|^{3}\right)+g_{0}(b)+\Sigma_{k=1} g_{k}(b) v^{k} \\
& =v-\frac{2}{3} \Lambda_{0} v^{2}+g_{0}(b)+\Sigma_{k=1} g_{k}(b) v^{k}+\mathcal{O}\left(|v|^{3}\right),
\end{aligned}
$$

where $g_{0}(b)=-2 b+\mathcal{O}\left(|b|^{2}\right)$ and $g_{k}(b)=\mathcal{O}(|b|)$. Observe that the function

$$
G(v, b)=P(v, b)-v=-\frac{2}{3} \Lambda_{0} v^{2}+g_{0}(b)+\Sigma_{k=1} g_{k}(b) v^{k}+\mathcal{O}\left(|v|^{3}\right),
$$

satisfy $G(0,0)=0$ and $\frac{\partial G}{\partial b}(0,0)=-2$. Then from the Implicit Function Theorem there exists a function

$$
b=g(v)=-\frac{1}{3} \Lambda_{0} v^{2}+\mathcal{O}\left(|v|^{3}\right),
$$

such that $G(v, g(v))=0$ for each $v \in(-\varepsilon, 0)$. In other words, for each $b$ sufficiently small with $b \Lambda_{0}<0$, there exists $v \in(-\varepsilon, 0)$ such that $P(v, g(v))=v$. That is, the unfolding (3) has a crossing limit cycle. Finally, to determine the stability of the limit cycle observe that

$$
\frac{\partial}{\partial v} P(v, b)=1-\frac{4}{3} \Lambda_{0} v+\Sigma_{k=1} k g_{k}(b) v^{k-1}+\mathcal{O}\left(|v|^{2}\right),
$$


and for each $b=g(v)$ with $v \in(-\varepsilon, 0)$

$$
\frac{\partial}{\partial v} P(v, g(v))=1-\frac{4}{3} \Lambda_{0} v+\mathcal{O}\left(|v|^{2}\right)=\left\{\begin{array}{ccc}
<1 & \text { if } & \Lambda_{0}<0 \\
>1 & \text { if } & \Lambda_{0}>0
\end{array}\right.
$$

5.1.2. Improper nodes. Assume $\lambda_{1}=\lambda_{2}$ and $\delta_{1}=\delta_{2}$. In this case

$$
S_{1}=0 \Leftrightarrow\left\{\begin{aligned}
e^{\lambda_{1} t_{1}} & =\frac{u}{v+t_{1}\left(\gamma_{2} r_{1}+\lambda_{1} v\right)}, \\
e^{\lambda_{1} t_{1}} & =\frac{\gamma_{2} r_{1}}{\gamma_{2} r_{1}-\lambda_{1} t_{1}\left(\gamma_{2} r_{1}+\lambda_{1} v\right)},
\end{aligned}\right.
$$

then $t_{1}=\frac{\gamma_{2} r_{1}(u-v)}{\left(\gamma_{2} r_{1}+\lambda_{1} u\right)\left(\gamma_{2} r_{1}+\lambda_{1} v\right)}$. That is

$$
S_{1}=0 \Leftrightarrow G_{1}(u, v)=\left(1+\tilde{\lambda}_{1} u\right) e^{\frac{1}{1+\tilde{\lambda}_{1} u}}-\left(1+\tilde{\lambda}_{1} v\right) e^{\frac{1}{1+\tilde{\lambda}_{1} v}}=0,
$$

where $\tilde{\lambda}_{1}=\frac{\lambda_{1}}{\gamma_{2} r_{1}}$. Similarly

$$
S_{2}=0 \Leftrightarrow\left\{\begin{aligned}
e^{\delta_{1} t_{2}} & =\frac{v+b}{u+b+t_{2}\left(r_{2}+\delta_{1}(u+b)\right)}, \\
e^{\delta_{1} t_{2}} & =\frac{r_{2}}{r_{2}-\delta_{1} t_{2}\left(r_{2}+\delta_{1}(u+b)\right)},
\end{aligned}\right.
$$

then $t_{2}=\frac{-r_{2}(u-v)}{\left(r_{2}+\delta_{1}(u+b)\right)\left(r_{2}+\delta_{1}(v+b)\right)}$. That is

$S_{2}=0 \Leftrightarrow G_{2}(u, v)=\left(1+\tilde{\delta}_{1}(-v-b)\right) e^{\frac{1}{1+\tilde{\delta}_{1}(-v-b)}}-\left(1+\tilde{\delta}_{1}(-u-b)\right) e^{\frac{1}{1+\tilde{\delta}_{1}(-u-b)}}=0$, where $\tilde{\delta}_{1}=-\frac{\delta_{1}}{r_{2}}$. As in the previous section

$$
G_{2}(u, v)=G_{1}(-(v+b),-(u+b)),
$$

and besides Lemma 1 is satisfied by $H(z)=\left(1+\tilde{\lambda}_{1} z\right) e^{\frac{1}{1+\tilde{\lambda}_{1} z}}$.

5.1.3. Assume $\lambda_{1} \neq 0, \lambda_{2}=0, \delta_{1} \neq 0, \delta_{2}=0$. Then

$$
S_{1}=0 \Leftrightarrow\left\{\begin{aligned}
e^{\lambda_{1} t_{1}} & =\frac{1+\tilde{\lambda}_{1} u}{1+\tilde{\lambda}_{1} v}, \\
e^{\lambda_{1} t_{1}} & =\frac{1+\tilde{\lambda}_{1} v+\lambda_{1} t_{1}}{1+\tilde{\lambda}_{1} v},
\end{aligned}\right.
$$

then $t_{1}=\frac{u-v}{\gamma_{2} r_{1}}$. That is

$$
S_{1}=0 \Leftrightarrow G_{1}(u, v)=\left(1+\tilde{\lambda}_{1} u\right) e^{-\tilde{\lambda}_{1} u}-\left(1+\tilde{\lambda}_{1} v\right) e^{-\tilde{\lambda}_{1} v}=0,
$$

where $\tilde{\lambda}_{1}=\frac{\lambda_{1}}{\gamma_{2} r_{1}}$. Similarly to the previous cases $S_{2}=0 \Leftrightarrow G_{2}(u, v)=0$, where $G_{2}$ satisfy $(9)$, and Lemma 1 is satisfied for $H(z)=\left(1+\tilde{\lambda}_{1} z\right) e^{-\tilde{\lambda}_{1} z}$. 
5.1.4. Assume $\lambda_{1}=\lambda_{2}=0, \delta_{1}=\delta_{2}=0$. Then $S_{1}=0 \Leftrightarrow u=h_{1}(v)=-v$, with $t_{1}=-\frac{2 v}{\gamma_{2} r_{1}}$, and $S_{2}=0 \Leftrightarrow v=h_{2}(u)=-2 b-u$, with $t_{2}=-\frac{2(u+b)}{r_{2}}$.

5.2. Focus-focus singularity: $r_{1}=0$ and $r_{2}=0$. In this case $\lambda_{1,2}=\alpha_{1} \pm i \beta_{1}$ and $\delta_{1,2}=\alpha_{2} \pm i \beta_{2}$. Then

$$
S_{1}=0 \Leftrightarrow\left\{\begin{aligned}
e^{\lambda_{1} t_{1}} & =e^{\lambda_{2} t_{1}} \\
u & =e^{\lambda_{1} t_{1}} v
\end{aligned}\right.
$$

that is, $t_{1}=\frac{\pi}{\beta_{1}}$ and $u=h_{1}(v)=-e^{\frac{\alpha_{1} \pi}{\beta_{1}}} v$. Therefore

$$
S_{2}=0 \Leftrightarrow\left\{\begin{aligned}
e^{\delta_{1} t_{2}} & =e^{\delta_{2} t_{2}} \\
v & =e^{\delta_{1} t_{2}} u+b\left(e^{\delta_{1} t_{2}}-1\right) .
\end{aligned}\right.
$$

That is $t_{2}=\frac{\pi}{\beta_{2}}$ and $v=h_{2}(u)=-e^{\frac{\alpha_{2} \pi}{\beta_{2}}} u-b\left(e^{\frac{\alpha_{2} \pi}{\beta_{2}}}+1\right)$. For each $\varepsilon>0$ we define the Poincaré map $P:(-\varepsilon, 0) \rightarrow(-\varepsilon, 0)$ given by

$$
P(v)=h_{2}\left(h_{1}(v)\right)=e^{\Lambda_{0} \pi} v-b\left(e^{\frac{\alpha_{2} \pi}{\beta_{2}}}+1\right),
$$

which for each $b$ such that $b \Lambda_{0}<0$ has the fixed point $\tilde{v}=\frac{b\left(e^{\frac{\alpha_{2} \pi}{\beta_{2}}}+1\right)}{e^{\Lambda_{0} \pi}-1}<0$, which is stable if $\Lambda_{0}<0$ and unstable if $\Lambda_{0}>0$.

\subsection{Invisible fold-focus singularity.}

5.3.1. Assume $r_{1}>0$ and $r_{2}=0$. From the previous cases we know that, $S_{1}=$ $0 \Leftrightarrow h_{1}(v)=-v+\lambda_{0} v^{2}-\lambda_{0}^{2} v^{3}+\cdots$, and $S_{2}=0 \Leftrightarrow h_{2}(u)=-e^{\frac{\alpha_{2} \pi}{\beta_{2}}} u-b\left(e^{\frac{\alpha_{2} \pi}{\beta_{2}}}+1\right)$. Then the Poincaré map is given by

$$
P(v, b)=h_{2}\left(h_{1}(v)\right)=e^{\frac{\alpha_{2} \pi}{\beta_{2}}}\left(v-\lambda_{0} v^{2}+\mathcal{O}\left(|v|^{3}\right)\right)-b\left(e^{\frac{\alpha_{2} \pi}{\beta_{2}}}+1\right) .
$$

Again we observe that the function

$$
\left.G(v, b)=P(v, b)-v=\left(e^{\frac{\alpha_{2} \pi}{\beta_{2}}}-1\right) v+\mathcal{O}\left(|v|^{2}\right)\right)-b\left(e^{\frac{\alpha_{2} \pi}{\beta_{2}}}+1\right),
$$

satisfy $G(0,0)=0$, and $\frac{\partial G}{\partial b}(0,0)=-\left(e^{\frac{\alpha_{2} \pi}{\beta_{2}}}+1\right) \neq 0$, then from the Implicit Function Theorem there is a function

$$
b=g(v)=c_{1} v+\mathcal{O}\left(|v|^{2}\right) \text { where } c_{1}=\frac{e^{\frac{\alpha_{2} \pi}{\beta_{2}}}-1}{e^{\frac{\alpha_{2} \pi}{\beta_{2}}}+1}=\left\{\begin{array}{ccc}
<0 & \text { if } & \alpha_{2}<0, \\
>0 & \text { if } & \alpha_{2}>0,
\end{array}\right.
$$

such that $G(v, g(v))=0$ for each $v<0$ sufficiently small. In other words, for each $b$ sufficiently small with $b \Lambda_{0}<0$, there exists $v<0$ such that $P(v, g(v))=v$. That is the unfolding (3) has a crossing limit cycle. Finally to determine the stability of the limit cycle we observe that

$$
\frac{\partial}{\partial v} P(v, b)=e^{\frac{\alpha_{2} \pi}{\beta_{2}}}\left(1-2 \lambda_{0} v+\mathcal{O}\left(|v|^{2}\right)\right)=\left\{\begin{array}{ccc}
<1 & \text { if } & \Lambda_{0}<0 \\
>1 & \text { if } & \Lambda_{0}>0
\end{array}\right.
$$

In this case the stability of the limit cycle only depends on the stability of the boundary focus. 
5.3.2. Assume $r_{1}=0$ and $r_{2}<0$. Then $S_{1}=0 \Leftrightarrow h_{1}(v)=-e^{\frac{\alpha_{1} \pi}{\beta_{1}}} v$, and $S_{2}=$ $0 \Leftrightarrow h_{2}(u)=-2 b-u-\delta_{0}(b+u)^{2}-\delta_{0}^{2}(b+u)^{3}+\cdots$. Then, the Poincaré map is given by

$P(v, b)=h_{2}\left(h_{1}(v)\right)=e^{\frac{\alpha_{1} \pi}{\beta_{1}}}(1+\mathcal{O}(|b|)) v+e^{\frac{2 \alpha_{1} \pi}{\beta_{1}}}\left(-\delta_{0}+\mathcal{O}(|b|)\right) v^{2}+\mathcal{O}\left(|v|^{3}\right)+g_{0}(b)$, where $g_{0}(b)=-2 b+\mathcal{O}\left(|b|^{2}\right)$. Again observe that the function

$$
G(v, b)=P(v, b)-v=-v+e^{\frac{\alpha_{1} \pi}{\beta_{1}}}(1+\mathcal{O}(|b|)) v+\mathcal{O}\left(|v|^{2}\right)+g_{0}(b),
$$

satisfy $G(0,0)=0$, and $\frac{\partial G}{\partial b}(0,0)=-2$, then from the Implicit Function Theorem there is a function

$$
b=g(v)=c_{1} v+\mathcal{O}\left(|v|^{2}\right), \text { where } c_{1}=\frac{1}{2}\left(e^{\frac{\alpha_{1} \pi}{\beta_{1}}}-1\right)=\left\{\begin{array}{cc}
<0 & \text { if } \quad \alpha_{1}<0, \\
>0 & \text { if } \quad \alpha_{1}>0,
\end{array}\right.
$$

such that $G(v, g(v))=0$ for each $v<0$ sufficiently small. In other words, for each $b$ sufficiently small with $b \Lambda_{0}<0$, there exists $v<0$ such that $P(v, g(v))=v$. That is, the unfolding (3) has a crossing limit cycle. Finally, to determine the stability of the limit cycle observe that

$$
\begin{aligned}
\frac{\partial}{\partial v} P(v, b) & =e^{\frac{\alpha_{1} \pi}{\beta_{1}}}(1+\mathcal{O}(|b|))+\mathcal{O}(|v|) \\
& =e^{\frac{\alpha_{1} \pi}{\beta_{1}}}(1+\mathcal{O}(|v|))+\mathcal{O}(|v|)=\left\{\begin{array}{lll}
<1 & \text { if } & \Lambda_{0}<0 \\
>1 & \text { if } & \Lambda_{0}>0
\end{array}\right.
\end{aligned}
$$

As in the previous case, the stability of the boundary focus determines the stability of the limit cycle. This completes the proof of Theorem 2.

\section{FINAL REMARKS}

We have established under which conditions a family of DPWL systems with a discontinuity line, which satisfy the generic condition of having a fold point in each zone, undergoes the pseudo-Hopf bifurcation. Although this phenomenon has been studied in several articles, it has always been in the context of searching for multiple crossing limit cycles, and as far as we know, a similar result had not been established previously.

\section{ACKNOWLEDGEMENTS}

The second author is partially supported by MINECO grants MTM2016-77278$\mathrm{P}$ and MTM2013-40998-P, an AGAUR grant number 2014SGR-568, and the grant FP7-PEOPLE-2012-IRSES 318999.

\section{REFERENCES}

[1] Akhmet M.U. Perturbations and Hopf bifurcation of the planar discontinuous dynamical system, Nonlinear Anal. 60 (2005), 163-178.

[2] Artés J.C., Llibre J., Medrado J.C. \& Teixeira M.A. Piecewise linear differential systems with two real saddles, Math. Comput. Simulation 95 (2013), 1-22.

[3] Buzzi C., Pessoa M. \& Torregrosa J. Piecewise linear perturbations of a linear center, Discrete Continuous Dyn. Syst. 33 (2013), 3915-3936. 
[4] Euzébio R.D. \& Llibre J. On the number of limit cycles in discontinuous piecewise linear differential systems with two pieces separated by a straight line, J. Math. Anal. Appl. 424 (2015), 475-486

[5] Filippov, A.F. Differential Equations with Discontinuous Right-hand Sides, Kluwer Academic Publishers, Dordrecht. 1988.

[6] Freire E., Ponce E. \& Torres F. Hopf-like bifurcations in planar piecewise linear systems, Publ. Mat. 41 (1997), 135-148.

[7] Freire E., Ponce E., Rodrigo F. \& Torres F. Bifurcation sets of continuous piecewise linear systems with two zones, Internat. J. Bifur. Chaos Appl. Sci. Engrg. 8 (1998), 2073-2097.

[8] Freire E., Ponce E. \& Torres F. Canonical Discontinuous Planar Piecewise Linear Systems, SIAM J. Appl. Dyn. Syst. 11 (2012), 181-211.

[9] Freire E., Ponce E. \& Torres F. Planar Filippov systems with maximal crossing set and piecewise linear focus dynamics, Progress and Challenges in Dynamical Systems, Springer, 2013, pp 221-232.

[10] Freire E., Ponce E. \& Torres F. A general mechanism to generate three limit cycles in planar Filippov systems with two zones, Nonlinear Dynam. 78 (2014), 251-263.

[11] Freire E., Ponce E. \& Torres F. On the critical crossing cycle bifurcation in planar Filippov systems, J. Differential Equations 259 (2015), 7086-7107.

[12] Guardia M., Seara T.M. \& Teixeira M.A. Generic bifurcations of low codimension of planar Filippov Systems, J. Differential Equations 250 (2011), 1967-2023.

[13] Guckenheimer J. \& Holmes P. Nonlinear oscillations, Dynamical systems, and bifurcations of vector fields, Applied Mathematical Sciences, 1993.

[14] Han M. \& Zhang W. On Hopf bifurcation in non-smooth planar systems, J. Differential Equations 248 (2010), 2399-2416.

[15] Huan S.M. \& Yang X.S. The number of limit cycles in general planar piecewise linear systems, Discrete Contin. Dyn. Syst. 32 (2012), 2147-2164.

[16] Huan S.M. \& Yang X.S. Existence of limit cycles in general planar piecewise linear systems of saddle-saddle dynamics, Nonlinear Anal. 92 (2013), 82-95.

[17] Huan, S.M. \& Yang X.S. On the number of limit cycles in general planar piecewise linear systems of node-node types, J. Math. Anal. Appl. 411 (2014), 340-353.

[18] Kuznetsov Yu.A., Rinaldi S. \& Gragnani A. One parameter bifurcations in planar Filippov systems, Internat. J. Bifur. Chaos Appl. Sci. Engrg. 13 (2003), 2157-2188.

[19] Kuznetsov Yu.A. Elements of Applied Bifurcation Theory, Springer-Verlag, 2004.

[20] Liping Li. Three crossing limit cycles in planar piecewise linear systems with saddle-focus type, Electron. J. Qual. Theory Differ. Equ. 70 (2014), 1-14.

[21] Llibre J., Ponce E. \& Torres F. On the existence and uniqueness of limit cycles in Liénard differential equations allowing discontinuities, Nonlinearity 21 (2008), 2121-2142.

[22] Llibre J. \& Ponce E. Three nested limit cycles in discontinuous piecewise linear differential systems, Dyn. Contin. Discrete Impuls. Syst. Ser. B Appl. Algorithms 19 (2012), 325-335.

[23] Llibre J., Teixeira M.A. \& Torregrosa J. Lower bounds for the maximum number of limit cycles of discontinuous piecewise linear differential systems with a straight line of separation, Internat. J. Bifur. Chaos Appl. Sci. Engrg. 23 (2013), 1350066-1-10.

[24] Llibre J., Novaes D.D., \& Teixeira M.A. Limit cycles bifurcating from the periodic orbits of a discontinuous piecewise linear differentiable center with two zones, Internat. J. Bifur. Chaos Appl. Sci. Engrg. 17 (2015), 1550144-1-11.

[25] Medrado J.C \& Torregrosa J. Uniqueness of limit cycles for sewing planar piecewise linear systems, J. Math. Anal. Appl. 431 (2015), 529-544.

[26] Simpson D.J.W. \& Meiss J.D. Andronov-Hopf bifurcations in planar, Piecewise-smooth, Continuous flows, Phys. Lett. A 371 (2007), 213-220.

[27] Simpson D.J.W. \& Meiss J.D. Unfolding a codimension two, Discontinuous, Andronov-Hopf bifurcation, Chaos 18 (2008), 033125.

1 Departamento de Matemáticas, Universidad de Sonora, Hermosillo, Mexico

E-mail address: verduzco@mat.uson.mx 
2 Departament de Matemàtiques, Universitat Autònoma de Barcelona, Bellaterra, 08193 Barcelona, Catalonia, Spain.

E-mail address: jllibre@mat.uab.cat 